Ann. Zootech., I979, 28 (2), I73-I84.

\title{
Evacuation gastrique de l'amidon et de l'azote d'un régime semi-purifié à base d'amidon de maîs chez le Porc
}

\author{
J.-C. CUBER et J.-P. IAPLACE \\ avec la collaboration technique de C. Girmain et P. TaIssade \\ Laboratoive de Physiologie de la Nutrition \\ Centre national de Rechevches Zootechniques, I.N.R.A., \\ 78350 Jouy-en-Josas, France.
}

\begin{abstract}
Résumé
Les cinétiques d'évacuation gastrique de l'amidon et de l'azote ont été étudiées, à la suite de l'ingestion d'un régime semi-purifié contenant 82 p. Ioo d'amidon de maïs et 7 p. roo de protéines, chez 3 porcs soumis à fistulation réentrante du duodénum proximal en vue de la collecte et de l'échantillonnage des digesta. L'azote a été dosé par la méthode Kjeldahl. L'amidon est mesuré sur la base de l'ensemble du glucose vrai $(<2 \mathrm{p}$. 10o) et des polymères $\alpha$ du glucose, de façon a pouvoir établir la relation entre évacuation gastrique et absorption quantitative des produits d'hydrolyse retrouvés dans la veine porte. La méthode utilisée, qui n'est en aucune façon un dosage de l'amidon sensu stricto a été développée au laboratoire par modification de la méthode de HoLz, et permet le traitement de 200 échantillons par jour.

L'évacuation gastrique de l'amidon et de l'azote, à la suite d'un repas apportant $800 \mathrm{~g}$ de M.S. après 24 heures de jeûne, présente comme celle de la M.S. une phase initiale, brève, très intense ( $23 \mathrm{p}$. I 00 de l'amidon et $28 \mathrm{p}$. I oo de l'azote ingérés, en $15 \mathrm{mn}$ ) puis un décours régulier plus lent. En 7 heures, 54 p. Ioo de l'amidon et l'équivalent de roo p. Ioo de l'azote ingéré sont collectés.

La quantité d'amidon émise par l'estomac est toujours très significativement corrélée à celle de la matière sèche, quelle que soit la quantité ingérée. La cinétique d'évacuation gastrique de l'amidon apparaît conme le principal facteur de contrôle de la cinétique d'apparition des sucres réducteurs dans la veine porte. La quantité d'azote évacuée par l'estomac est moins étroitement reliée à celle de matière sèche émise, et ce d'autant moins longtemps que la quantité ingérée est plus faible. L'importance relative de l'évacuation apparente d'azote résuite de l'addition d'azote endogène d'une part, et d'une évacuation différentielle plus rapide de l'azote d'autre part.
\end{abstract}

\section{Introduction}

Dans le but de préciser les relations entre les fonctions digestives de transit, de digestion enzymatique et d'absorption, nous avons précédemment décrit (CUBER et LAPIACE, I 979) l'évacuation gastrique de la matière sèche chez le Porc 
pour un régime semi-purifié à base d'amidon de maïs, dont sont connus par ailleurs les effets sur la motricité de l'intestin grêle (LAPLACE, 1978) et l'absorption (RERAT et al., I974; RERAT, I975a). Nous apportons ici, pour ce même régime, la description de la cinétique d'évacuation gastrique de l'amidon et de l'azote, afin d'en évaluer l'importance à l'égard de la cinétique de mise à disposition des nutriments.

\section{Matériel et méthodes}

Trois porcs de race Large White, d'un poids vif compris entre 55 et $65 \mathrm{~kg}$ ont été préparés (fistule réentrante du duodénum proximal) et entretenus en cage selon LAPLACE et TOMASSONE (I970). Ils ont reçu exclusivement, au cours d'une période préexpérimentale de 8 jours et au cours de la période expérimentale, un aliment composé de 82 p. Ioo d'amidon de maïs, 3 p. Ioo de cellulose purifiée et I5 p. Ioo d'un complément azoté (LAPLACE, I978). Ce régime , apportant $7 \pm 0,06 \mathrm{p}$. roo de protéines ( $\mathrm{N} \times 6,25$ - I I échantillons) a été le plus souvent distribué sous forme de soupe (poids de farine fraîche /eau, I : I) une fois par jour à $9 \mathrm{~h}$. Chaque repas d'épreuve est alors précédé d'un " jeûne " de $24 \mathrm{~h}$. Dans un certain nombre de cas, un repas supplémentaire apportant $440 \mathrm{~g}$ de matière sèche aut total a été fourni à $2 \mathrm{I}$ h la veille d'une épreuve de collecte.

En somme, les repas d'épreuve dits "après 24 h de jeûne " sont les repas usuels auxquels l'animal est parfaitement habitué, tant du point de vue composition de l'aliment que du point de vue rythme de distribution (chaque jour à $9 \mathrm{~h}$ ). Le jour de distribution d'un repas supplémentaire à $2 \mathrm{I}$ h, l'épreuve usuelle avec contrôle durant $7 \mathrm{~h}$ est achevée avant la seconde distribution, et l'épreuve du lendemain à $9 \mathrm{~h}$ constitue $u$ n repas dit "après $\mathrm{I} 2 \mathrm{~h}$ de jeûne ". Cette dernière situation n'a jamais été appliquée durant 2 ou plusieurs jours consécutifs, et ne fait donc pas, à la différence des épreuves après 24 h de jeûne, l'objet d'une accoutumance de l'animal.

Les effluents gastro-duodénaux sont recueillis, échantillonnés et restitués à 1'animal selon une procédure semi-automatique (BORGIDA et L,APLACE, I977) simplifiée (suppression de l'enregistrement - contrôle de la réintroduction). Au cours des $7 \mathrm{~h}$ postprandiales, les digesta émis dans un bécher placé à la glace fondante sont collectés par période de $5 \mathrm{mn}$ jusqu'à la quinzième minute, de $\mathrm{I} 5 \mathrm{mn}$ jusqu'au temps $t=\mathrm{I} \mathrm{h}$, de $30 \mathrm{mn}$ ensuite. Leur poids frais est enregistré et une aliquote de chaque collecte de digesta soumis à une agitation vigoureuse, est prélevée à la pompe. Le reste est restitué à l'animal après réchauffement. L'aliquote pesée est acidifiée à $\mathrm{pH} 2$ avec l'acide chlorhydrique ( $\mathrm{HCl}-6 \mathrm{~N}$ ) pour inactiver Jes amylases salivaire et pancréatique, puis neutralisée avec de la soude normale $(\mathrm{NaOH}-\mathrm{IN})$, congelée et lyophylisée. La matière sèche est déterminée après $24 \mathrm{~h}$ à 1'étuve à $104^{\circ} \mathrm{C}$.

L'azote a été dosé par la méthode de KJELDHAL. La détermination de l'amidon a été effectuée selon la méthode de HoLz (I977) modifiée au Iaboratoire. En effet, la technique de HoLz (I977) conçue pour doser l'amidon dans les céréales et farines de céréales, comporte l'adjonction au milieu réactionnel de tampon tris et d'éthylène glycol, qui éviterait l'interférence du glucose et du maltose dans le dosage. Dans notre cas, le dosage du glucose vrai effectué par la méthode de HIL, et KESSLER (I G6I), sur 3 échantillons d'aliment et sur I7 échantillons de contenus digestifs prélevés aux divers temps de notre chronologie de collecte, a montré que 


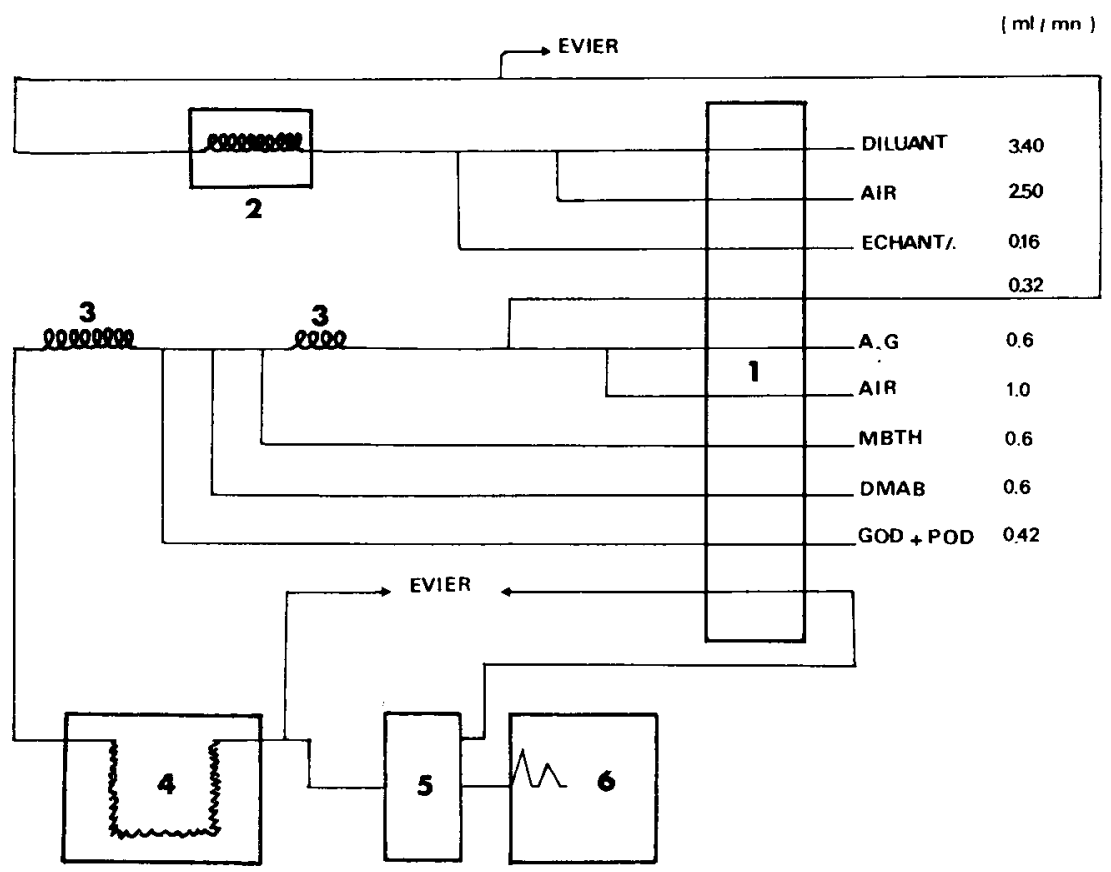

FIG. I. - Schéma du circuit analytique utilisé pour le dosage global du glucose et de ses polymères $\alpha$ $\mathbf{I}=$ Pompe péristaltique; $2=$ Réfrigérant $\left(20{ }^{\circ} \mathrm{C}\right) ; 3=$ Bobines mélangeuses; $4=$ Bainmarie $\left(3^{\circ}{ }^{\circ} \mathrm{C}\right) ; 5=$ Colorimètre à $620 \mathrm{~nm} ; 6=$ Envegistreur.

A.G. : anyloglucosidase; M.B.T.H. : Hydrazono - 2 - dihydro-2,3 méthyl 3 benzothiazole chlorhydrate pur (Touzart Matignon).

D.M.A.B. : Acide 3 - Dimethylaminobenzoïque à 99 p. Ioo (Aldrich Chem Co).

G.O.D. : glucose oxydase type 2 (Sigma Chem. Co).

P.O.D. : peroxydase type 2 (Sigma Chem. Co).

Scheme of the analytical circuit used for the overall determination of glucose and its $\alpha$ polymers. I $=$ Peristaltic pump; $2=$ cooling agent $\left(20{ }^{\circ} \mathrm{C}\right) ; 3=$ miscing coils; $4=$ water bath; $5=$ colorimeter at $620 \mathrm{~nm} ; 6=$ recording apparatus.

A.G. : amyloglucosidase; M.B.T.H. : pure hydrazono-2-dihydro-2,3 methyl 3 benzothiazole chlorhydrate (Touzart Matignon).

D.M.A.B. : 99 p. Ioo 3 Dimethylaminobenzoic acid (Aldrich Chem. Co).

G.O.D. : glucose oxydase type 2 (Sigma Chem. Co).

P.O.D. : peroxydase type 2 (Sigma Chem. Co.).

l'aliment utilisé et les digesta collectés et stabilisés comme il a été décrit (CUIBER et LAPLACE, 1979) contiennent toujours moins de 2 p. Ioo de glucose. Par ailleurs, recherchant la relation entre évacuation gastrique et absorption des produits de la digestion, il a été choisi de définir pour ce travail le dosage de l'amidon comme se rétérant au glucose ( $\because 2$ p. Ioo) et à tous les polymères a du glucose. Ceci permet l'évaluation globale de l'amidon et des produits de son hydrolyse qui auraient pu se former dans les io premiers cm du duodénum ou lors de la collecte des digesta.

Pour ces raisons, l'adjonction de tampon tris et d'éthylène glycol a été supprimée. La solubilisation de l'amidon dans le diméthyl sulfoxyde selon HoLz (1977) a été maintenue. Le circuit analytique sur chaîne Technicon Autoanalyzer I a été modifié (fig. I) sur 2 points : automatisation de la dilution au taux défini par Holz (I977) de l'échantillon solubilisé, et substitution à la solution de rinçage 
utilisée par HoLz (I977) de la solution utilisée par ailleurs pour la dilution de l'échantillon solubilisé. Ceci permet de couvrir l'échelle o à roo p. roo au lieu de 50 à Ioo p. Ioo dans le cas de HoLz (1977). Enfin, nous avons utilisé, à la différence de Holz (I977), une amylogltucosidase d'Aspergillus niger (NOVO Pharmacentique) spécifique des liaisons $\alpha$ I-4 et $\propto$ I-6, pour hydrolyser l'amidon dans le circuit analytique. La non dégradation de la cellulose purifiée (présente dans le régime) par cette enzyme a été vérifiée.

La technique ainsi modifiée autorise le traitement de 200 échantillons par jour. Sa validité a été éprouvée par comparaison avec la technique de Thivend, Mercier et Guiriot (I965). L'écart entre les teneurs mesurées par l'une et l'autre méthodes (dosages en triple de 6 échantillons de digesta), dans un sens ou dans l'autre, est compris entre $\mathrm{r}, \mathrm{O}$ et $4,6 \mathrm{p}$. Ioo.

\section{Résultats}

\section{I. - Cinétique d'évacuation de l'amidon et de l'azote}

(repas d'épreuve après 24 h de jeûne : $880 \mathrm{~g}$ de M.S., $720 \mathrm{~g}$ d'amidon, 9,9 g d'azote)

I.es courbes (fig. 2) exprimant respectivement les quantités cumulées d'amidon ou d'azote évacuées par l'estomac, montrent, comme c'était le cas pour la matière sèche, une première période brève d'évacuation gastrique intense conduisant à la récupération en $15 \mathrm{mn}$ de $23 \mathrm{p}$. roo de 1'amidon et $28 \mathrm{p}$. Ioo de l'azote ingérés (moyenne de II épreuves au total chez 3 porcs). L'évacuation de ces 2 constituants se dérotıle ensuite beatucoup plus lentement et de façon régulière, amenant après 7 heures de collecte à la récupération de 53,9 p. Ioo de l'amidon ingéré, et d'une quantité d'azote analogue à celle ingérée $(98,7$ p. IOO).

Sur l'ensemble des collectes réalisées chez 3 porcs, la quantité d'amidon recueillie à chaque temps de la chronologie est très significativement corrélée à la quantité correspondante de matière sèche $(p<0,0 \mathrm{I})$ quoique la valeur de $r$ dimintue de 0,997 à 0,909 entre $5 \mathrm{mn}$ et $6 \mathrm{~h}$. Ce même cofficient $r$ est de $0,7 \mathrm{I} 2$ $(p<0,05)$ à la septième heure. Dans le cas des quantités d'azote, la corrélation avec les quantités de matière sèche est significative durant 5 heures $(r$ de 0,955 à 0,822 à la quatrième heure $-p<0, \mathrm{CI}$, et $r=0,67 \mathrm{I}-p<0,05$ à la cinquième heure). Cette corrélation est ensuite totalement absente.

I, a variabilité (cœefficient de variation) de la récupération de l'amidon et de l'azote d'une épreuve à l'autre chez un même Porc évolue dans le temps de façon analogue à celle de la matière sèche : respectivement pour l'amidon et l'azote, 23 et I $7 \mathrm{p}$. Ioo au temps $t=30 \mathrm{mn}$, I 7 et II p. Ioo au temps $t=2 \mathrm{~h}, 8$ et $6 \mathrm{p}$. Ioc au temps $t=7 \mathrm{~h}$. La variabilité entre pores (fig. 2) est un peu plus importante dans les premières heures : 27 et $23 \mathrm{p}$. Ioo au temps $t=30 \mathrm{mn}, 20, \mathrm{I}$ et $\mathrm{Ig}, 8 \mathrm{p}$. IoO au temps $t=2 \mathrm{~h}$ respectivement pour l'amidon et l'azote.

\section{2. - Influence de la quantité ingérée}

Chez un même porc (fig. 3), les cinétiques d'évacuation de l'amidon lors de repas d'épreuve de $880 \mathrm{~g}$ de matière sèche ( $720 \mathrm{~g}$ d'amidon - $9,9 \mathrm{~g}$ d'azote), I $320 \mathrm{~g}$ de matière sèche (I o80 g - I 4,8 g) et I $760 \mathrm{~g}$ de matière sèche (I 440 g - I $9,8 \mathrm{~g}$ ) après 


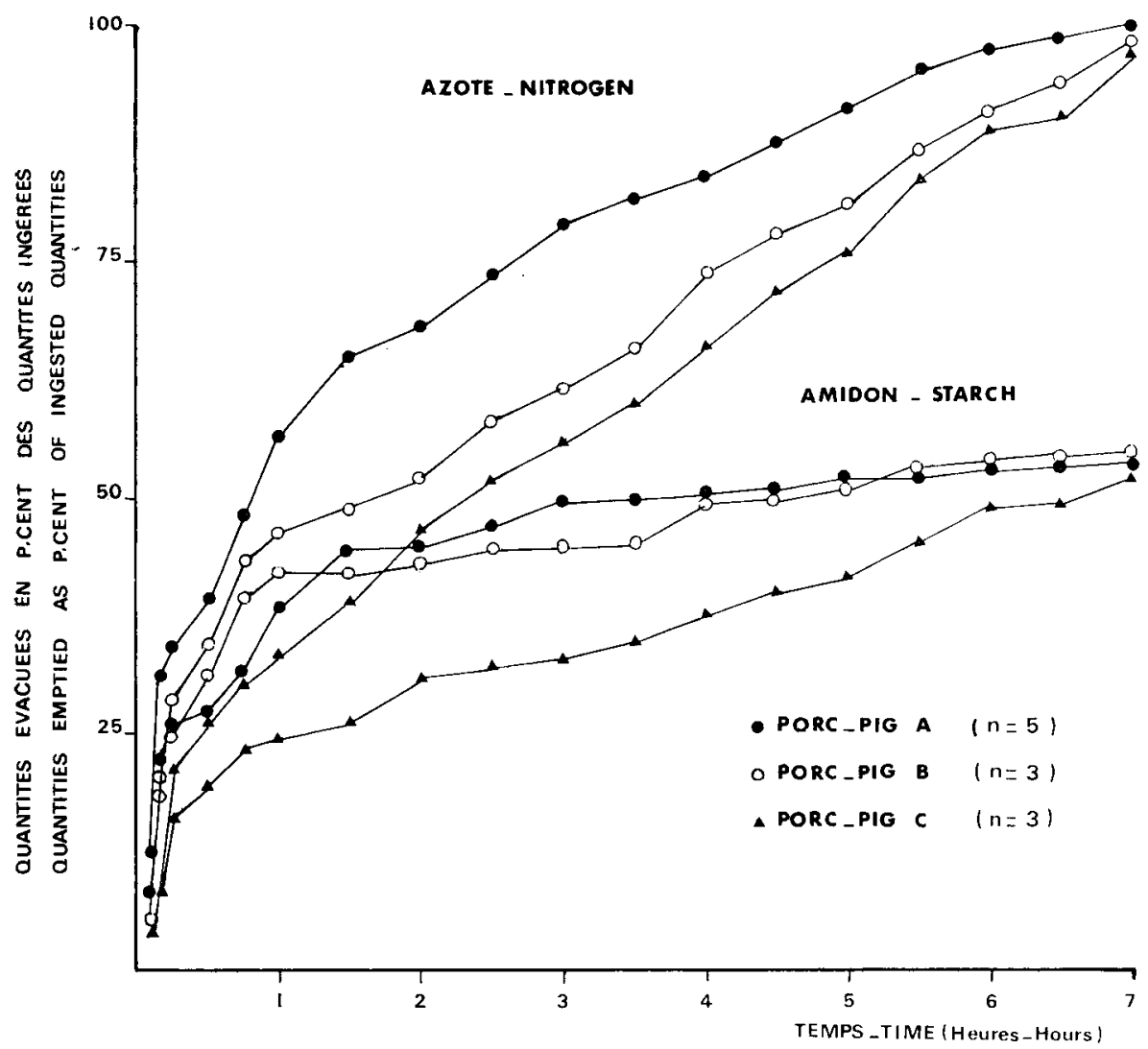

FIG. 2. - Evacuation gastrique de l'amidon et de l'azote chez le Porc apvès un repas (88o g de M.S., $720 \mathrm{~g}$ d'amidon, $9,9 \mathrm{~g}$ d'azote) distribué à 9 heuves. Courbes moyennes représentatives de l'évacuation gastrique chez trois porcs $A, B$ et $C$. Expression par cumul au fil du temps des quantités émises en p. roo des quantités ingérées.

Starch and nitrogen gastric emptying in the pig alter a test-meal $(880 \mathrm{~g}$ dry matter, $720 \mathrm{~g}$ starch, $9,9 \mathrm{~g}$ nitrogen) given at 9 a.m. Mean representative curves of gastric emptying in 3 pigs $A, B$ and $C$. Cumulative expression with time of the amounts emptied in $p$. Ioo of the ingested amounts.

$24 \mathrm{~h}$ de jeûne, montrent que cette évacuation est toujours, à tous les temps de la chronologie, très significativement $(p<0,0 I)$ corrélée à celle de la matière sèche $(r=0,9980$ et $r=0,9986$ à la septième heure, respectivement après les repas de I 320 et I $750 \mathrm{~g}$ de matière sèche). La quantité d'amidon collectée au total, respectivement pour les repas de 880 - I 320 et I $760 \mathrm{~g}$ de matière sèche, représente effectivement un pourcentage analogue de l'ingéré dans les 3 cas durant les 2 premières heures d'évacuation (I9,6-20,0 ei $20,6 \mathrm{p}$. Ioo au temps $t=30 \mathrm{mn}$ et $30,6-3 \mathrm{I}, 2$ et $34,3 \mathrm{p}$. roo au temps $t=2 \mathrm{~h}$ dans le cas de la figure 3 par exemple). Cependant, au bout de $7 \mathrm{~h}$, la proportion d'amidon récupéré décroît $(53,6-48$, I et 42,6 p. roo) lorsqu'augmente la quantité ingérée.

Dans le cas de l'azote, l'absence de corrélation à la sixième et à la septième heure pour un repas de $880 \mathrm{~g}$ (M.S.) disparaît au profit d'une relation significative $(p<0$, Io) pour un repas de I $320 \mathrm{~g}$, et d'une corrélation très significative dans 


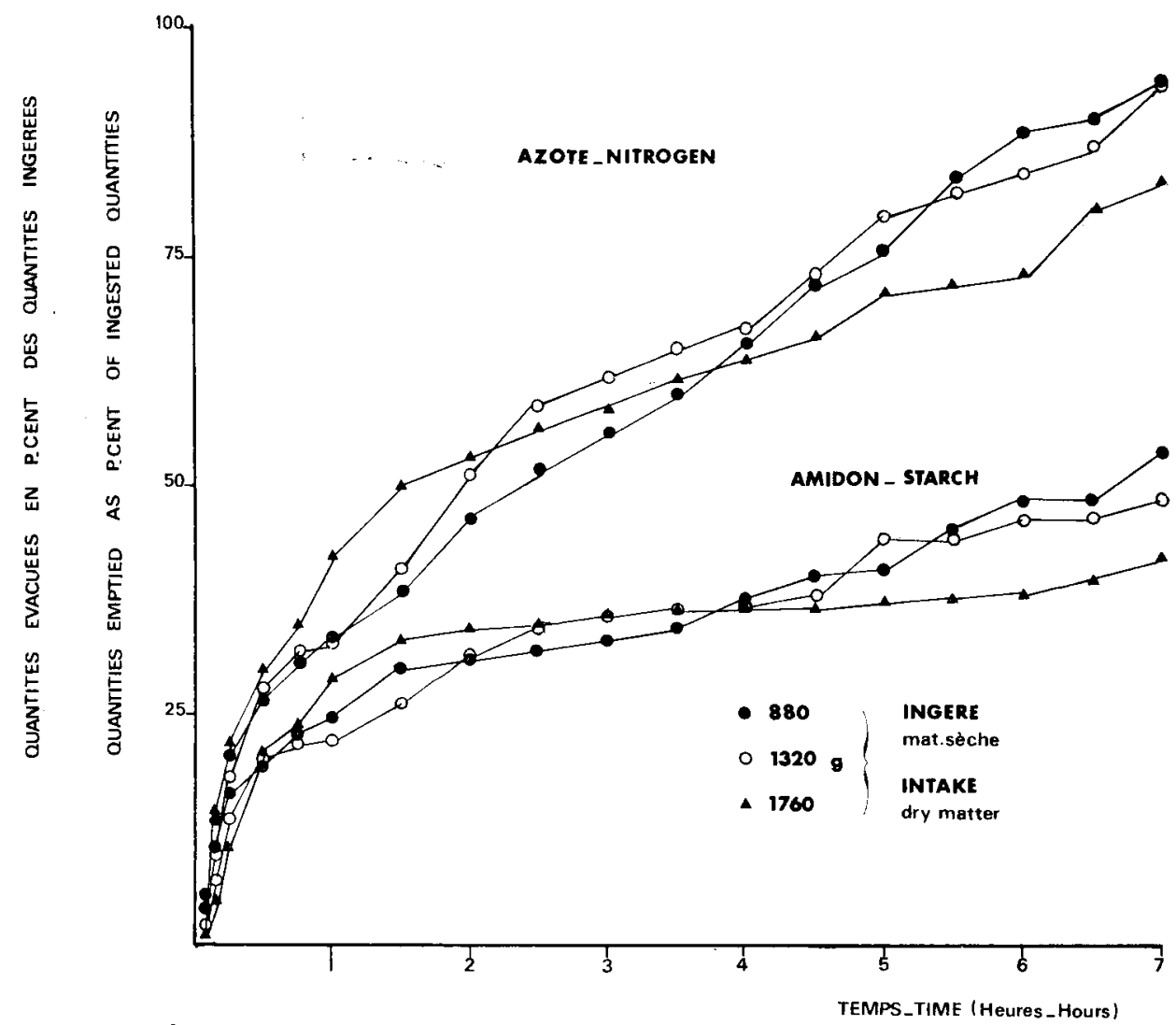

FIG. 3. - Évacuation gastrique de l'amidon et de l'azote chez le Porc après un vepas d'épreuve. distribué à 9 heures. Courbes moyennes représentatives chez le même Porc C, selon le cas après ingestion de $880 \mathrm{~g}$ de M.S. (3 essais), de I $320 \mathrm{~g}$ de M.S. ( 1 o8o g d'amidon - I4,8 g d'azote 3 essais) ou de $1760 \mathrm{~g}$ de M.S. ( $1440 \mathrm{~g}$ d'amidon - $19,8 \mathrm{~g}$ d'azole - 2 essais). Expression par cumul au fll du temps des quantités émises en p. Ioo des quantités ingérées.

Starch and nitrogen gastric emptying in the pig after a test-meal given at 9 a.m. Mean representative curves in the pig C, either after ingestion of $880 \mathrm{~g}$ dry mater ( 3 trials), of I $320 \mathrm{~g}$ dry matter ( 1 o8o g starch - $14,8 \mathrm{~g}$ nitrogen - 3 trials) or of $1760 \mathrm{~g}$ dry matter ( $1440 \mathrm{~g}$ starch19,8 g nitrogen - 2 trials). Cumulative expression with time of the amounts emptied in $p .100$ of the ingested amounts.

le cas d'un repas de I $760 \mathrm{~g}(r=0,9989-p<0$,oI à la septième heure). Néanmoins, la quantité d'azote collectée en $7 \mathrm{~h}$, équivalente à celle ingérée pour un repas de $880 \mathrm{~g}$ de matière sèche, tend à décroître en valeur relative lorsqu'augmente la quantité ingérée. Les valeurs enregistrées dans le cas de la figure 3 sont, respectivement pour les repas de $880-\mathrm{I} 320$ et I $760 \mathrm{~g}$ de matière sèche, de 94,4-93,4 et 83 , I p. IoO.

\section{3. - Infuence de la durée du jềne préalable}

Lors de la distribution d'un même repas d'épreuve (88o g de matière sèche) $\mathrm{r} 2 \mathrm{~h}$ seulement après ingestion de $440 \mathrm{~g}$ de matière sèche (fig. 4) on ne constate 


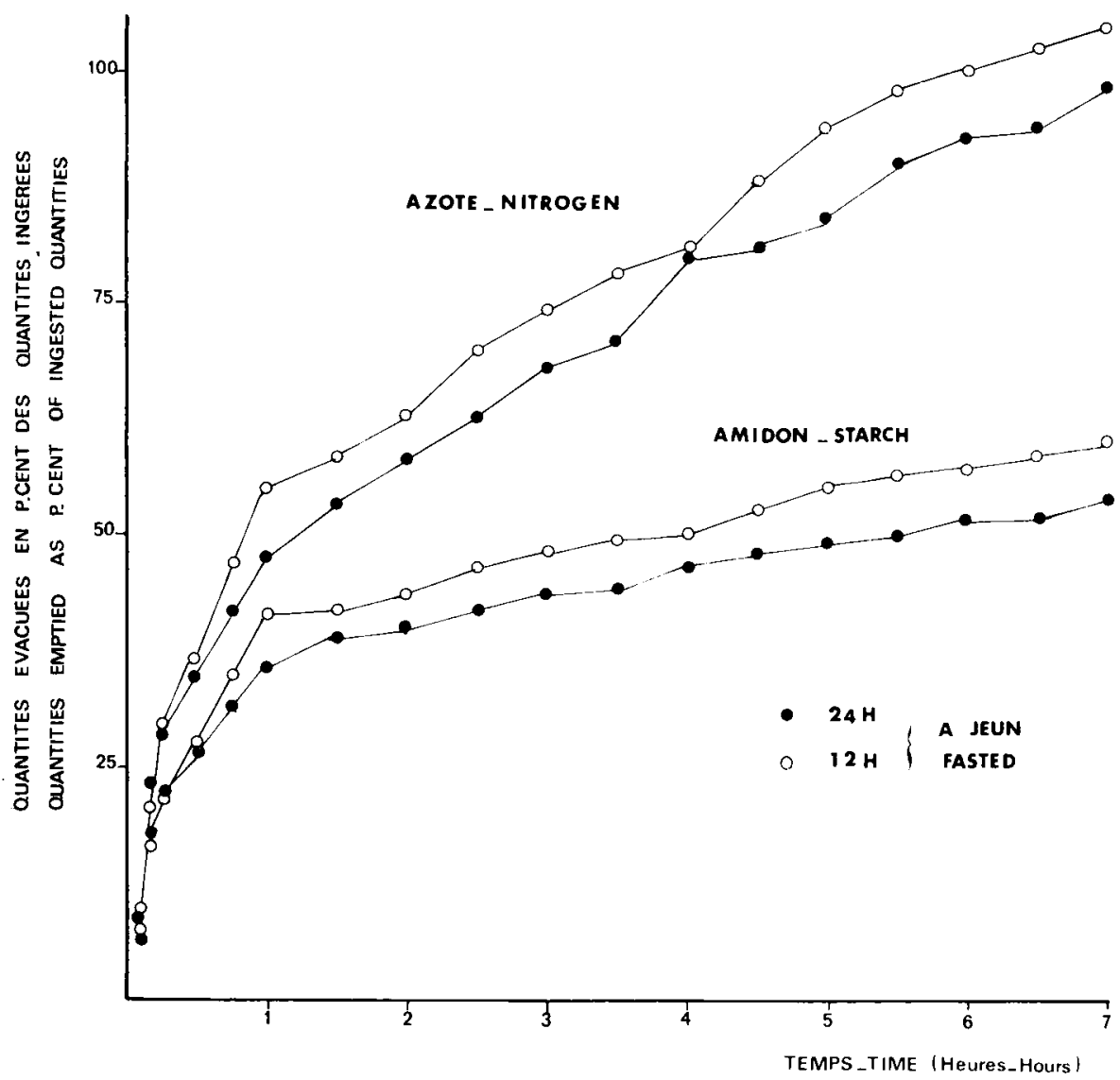

FIG. 4. - Courbes moyennes représentatives de l'évacuation gastrique de l'amidon et de l'azote après un repas de $880 \mathrm{~g}$ de M.S., selon le cas après $2+$ heures de jeûne (II essais de 3 porcs) ou 12 heures après ingestion de $44^{\circ} \mathrm{g}$ de M.S. (9 essais de 3 porcs). Expression par cumul au fl du temps des quantités émises en p. Ioo des quantités ingévées.

Mean representative curves of starch and nitrogen gastric emptying after a test-meal of $880 \mathrm{~g}$ dry matter, either a/ter 24 hours fasting (II trials with 3 pigs) or 22 hoursafteringestion of $440 \mathrm{~g}$ dry matter (9 trials with 3 pigs). Cumulative expression with time of the amounts emptied in $p$. Ioo of the amounts ingested.

aucune modification de la phase initiale rapide d'évacuation pendant les ${ }_{5} 5$ premières minutes. Par la suite, la réduction de la durée du jeûne à $\mathrm{I} 2 \mathrm{~h}$ a pour conséquence 1'évacuation de $42 \mathrm{~g}$ d'amidon supplémentaire à $60 \mathrm{mn}$, et $48 \mathrm{~g}$ au total à $1 \mathrm{a}$ septième heure.

La quantité totale damidon récupérée en 7 h dans cette situation équivaut alors à $60,3 \mathrm{p}$. Ioo ( $n=9$ épreuves) de la quantité d'amidon ingérée lors du repas d'épreuve au lieu de 53,9 p. Ioo ( $n=$ I I épreuves) après $24 \mathrm{~h}$ de jeûne. L'évacuation d'azote est également augmentée de $0,78 \mathrm{~g}$ à $60 \mathrm{mn}$ et $\mathrm{I}, 23 \mathrm{~g}$ au total à $1 \mathrm{a}$ septième leure, soit une récupération en $7 \mathrm{~h}$ équivalant à III p. Ioo $(n=9$ épreuves) de 1'azote ingéré au lieu de 98,7 p. roo $(n=$ I I épreuves), lors des repas après $24 \mathrm{~h}$ de jeûne. 


\section{Discussion}

Pour cette première tentative d'étude des relations entre l'évacuation gastrique et l'absorption des produits de la digestion, nous nous sommes limités à une approche très globale reposant sur l'analyse de l'évacuation de l'azote et des polymères du glucose issus de l'amidon apporté par le régime. Il importe de souligner à nouveau que la technique, dite par commodité "technique de dosage de l'amidon ", élaborée par modification de la technique de Holz (I977), n'est en aucune façon un dosage de l'amidon sensu stricto, mais seulement un dosage global de l'ensemble constitué par l'amidon et les produits de son hydrolyse. Cette procédure, dont l'avantage le plus évident réside dans le nombre considérable d'échantillons traités par jour, ne doit par ailleurs en aucun cas être généralisée arbitrairement. Sa validité a été testée dans le cas précis du régime à base d'amidon de maïs purifié et des effluents gastriques à la suite de son ingestion. Elle devra être réévaluée pour toute autre source amylacée. Ces réserves importantes étant faites, elle fournit une base de comparaison commode relativement aux données quantitatives d'absorption des glucides après ingestion d'amidon de maïs, telles qu'apportées par RERAT et al. (I974) et RERAT (I975a).

Cela étant, plusieurs points doivent être considérés. Le glucose est présent dans les digesta collectés à une concentration analogue à celle mesurée dans l'aliment, soit dans tous les cas moins de $2 \mathrm{p}$. IOo. Il est donc plausible qu'au site de collecte choisi et dans les conditions techniques utilisées pour la préparation des échantillons, le glucose présent soit d'origine alimentaire directe. Cette observation concorde avec celle de Holmes, BAyLEy et HorNey (r974) selon lesquels, en $\mathrm{I} 2 \mathrm{~h}$ de collecte, le glucose représente au plus I,4 p. Ioo de la quantité totale de matière sèche des digesta et $4 \mathrm{p}$. Ioo de l'amidon évacué par l'estomac. On peut aussi envisager l'hypothèse d'une absorption quasi immédiate du glucose au fur et à mesure de sa libération dans le duodénum proximal; il en résulterait les taux très faibles mentionnés ci-dessus. Cette hypothèse est utilisée par HOLMES, BAYLEY et HORNEY (I974) pour expliquer le fait qu'ils ne collectent en I 2 h que 47 à $59 \mathrm{p}$. IOO de la quantité d'amidon ingérée. Ils supposent donc une intense hydrolyse avec absorption immédiate en amont de la canule proximale.

Sur ce point, KEYS et DE BARTHE (I974) soulignent le contraste entre la digestibilité apparente de l'amidon de maïs (72 p. Ioo) dosé selon MAC RAE et ArMStrong (I968), de l'amylose (94 p. I00) et de l'amylopectine (66 p. I0o) au niveau duodénal et celle de la matière sèche (4 p. I0o). Ce fait, attribué par KEYs et DE BARTHE (r974) à l'action de l'amylase salivaire, tend à montrer que s'il y a effectivement attaque importante de l'amidon, l'hydrolyse n'est pas au niveau duodénal suffisamment avancée pour conduire à une importante disparition de matière sèche par absorption comme le supposaient HOLMES, BAYLEY et HoRNEY (I974). Par conséquent les faibles quantités de glucose observées dans le duodénum sont très certainement apportées par l'aliment, et l'amidon de maïs est pour l'essentiel digéré (i.e. absorbé) en aval du site de fistulation duodénale. Par ailleurs, à devx reprises, l'enregistrement de l'évacuation gastrique, à la suite d'un repas de $880 \mathrm{~g}$ de matière sèche après $24 \mathrm{~h}$ de jeîne, a été prolongé durant $24 \mathrm{~h}$. Dans ces deux cas, la quantité d'amidon récupérée en $\mathrm{I} 2 \mathrm{~h}$ a représenté 56,6 et 59,3 p. Ioo de l'amidon ingéré, en parfait accord avec les valeurs de HoLmES, BAYLEY et Horney (I974), soit 47 à $59 \mathrm{p}$. Ioo. Mais en $24 \mathrm{~h}$ ce sont 72,7 et 8o,9 p. xoo de l'amidon ingéré qui ont été retrouvés ce qui démontre que l'amidon non récupéré 
en $\mathrm{I} 2 \mathrm{~h}$ n'a pas en totalité disparu par absorption comme l'avaient supposé Hor,MEs, BAYIEY et HORNEY (I974).

Cependant, l'hypothèse d'une hydrolyse enzymatique avec absorption très rapide de glucose, intervenant l'une et l'autre dans les ro premiers centimètres du duodénum, n'est pas la seule qui puisse être envisagée. En effet, la quantité de matière sèche collectée en $24 \mathrm{~h}$, à la suite d'un repas de $880 \mathrm{~g}$ de matière sèche après $24 \mathrm{~h}$ de jeûne, ne représente que 95,9 à 97,0 p. roo de la quantité ingérée. Si l'on tient compte d'un certain apport endogène de matière sèche, ces observations suggèrent que l'évacuation gastrique du repas d'épreuve n'est pas totalement achevée. Mais, étant donné la régularité du rythme d'alimentation, cette fraction non évacuée du repas d'épreuve devrait être compensée dans la collecte par l'évacuation additionnelle du reliquat du repas distribué $24 \mathrm{~h}$ avant l'épreuve. Il faut donc bien admettre qu'une petite quantité de matière sèche disparaît effectivement au niveau le plus proximal. Ce fait ne peut trouver d'autre explication que celle fournie par l'importance des fermentations survenant dans 1'estomac, démontrée par les quantités d'acides organiques et en particulier d'acide lactique (FRIEND, Cunningham et Nichol,son, i963; Argenzio et Southworth, I975; Cimmfins, STEVENS et SOUTHWORTH, I975). Les quantités considérables d'acide lactique $(35 \mathrm{~g})$ apparaissant dans le sang de la veine porte (RERA' $\mathrm{T}$, I975a) en $8 \mathrm{~h}$ après ingestion d'environ $600 \mathrm{~g}$ d'amidon de mais et la précocité de cette apparition (avant que le gros intestin ne soit atteint par l'aliment de l'épreuve) témoignent sans doute, non seulement d'une inétabolisation de certains glucides dans la paroi digestive, mais aussi de l'importance de la fermentation lactique de l'amidon de maïs dans 1'estomac.

Enfin, s'il paraît vraisemblable qu'une certaine fraction de la matière sèche ait disparu par absorption des produits de sa fermentation, il reste douteux que ce mécanisme puisse rendre compte de la totalité du pourcentage de l'amidon ingéré qui n'est pas récupéré en 24 heures. Selon les données de RERP T (I975b) ce n'est qu'au-delà de 22 h après le repas que s'estompe la différence porto-artérielle des concentrations de sucres réducteurs. Néanmoins, cette phase terminale se traduit par l'absorption de $\mathrm{I} 5 \mathrm{~g}$ de glucides dans la période située entre 22 et $29 \mathrm{~h}$ après le repas. Ceci reste vrai, quelle que soit la quantité ingérée. D'une part, son accroissement accélère la phase initiale d'évacuation gastrique rapide (accroissement proportionnel des quantités émises) mais ne fait que prolonger l'évacuation ultérieure sans en modifier sensiblement le rythme, et d'autre part, le cœefficient d'absorption n'est pas affecté par l'augmentation du niveau d'ingestion (RERAT, I975a).

Les quantités de sucres réducteurs apparues dans le sang de la veine porte (RERAT, VAISSADE et VAUGEIADE, I978) à la suite de l'ingestion d'un repas apportant $720 \mathrm{~g}$ d'amidon de maïs (base matière sèche) sont, exprimées en p. Ioo de l'ingéré amidon, de $13-28-38$ et $45 \mathrm{p}$. Ioo respectivement $2,4,6$ et $8 \mathrm{~h}$ après le repas. Pour un repas identique du même régime, les quantités évacuées par 1'estomac représentent $40,46,52$ et $54 \mathrm{p}$. Ioo de l'amidon ingéré au bout de respectivement $2,4,6$ et $7 \mathrm{~h}$. Il est remarquable que les $40 \mathrm{p}$. Ioo évacués par l'estomac dans les deux premières heures ne produisent qu'une absorption de I3 p. Ioo dans le même temps. Ce décalage ne peut être attribué à un retard artificiellement créé par le fait de la collecte et de l'échantillonnage. Indépendamment de la fiabilité de leur réalisation, l'importance même de l'écart exclut cette hypothèse. Ceci suggère donc que le processus de digestion de l'amidon n'est pas aussi rapide qu'on aurait pû le supposer. La réduction progressive de cet écart peut être reliée à la progression des digesta dans l'intestin grêle et donc à la mise en jeu graduelle des surfaces absorbantes disponibles dans leur totalité. De fait, au-delà des premières 
heures, la similitude des cinétiques d'évacuation gastrique et d'absorption est frappante.

En somme, l'absorption par elle-même n'est pas le facteur limitant de l'apparition des sucres réducteurs dans la veine porte (RERAT, I 975a); la capacité d'hydrolyse enzymatique ne paraît pas en cause dans la mesure où, au-delà des 2 premières heures post-prandiales, la cinétique d'absorption reflète la cinétique d'évacuation gastrique. Celle-ci apparaît donc comme le véritable facteur contrôlant le rythme de mise à disposition de l'organisme des nutriments glucidiques.

En ce qui concerne l'azote, le phénomène le plus marquant quelle que soit la situation expérimentale mise en ouvre est la rapidité avec laquelle est recueillie une quantité équivalente à celle ingérée, relativement à l'évacuation de l'amidon ou globalement de la matière sèche. Ce phénomène peut résulter de 2 causes, dont l'une est d'évidence l'addition à l'azote exogène de la ration d'une quantité non négligeable d'azote endogène, et dont l'autre tient aux facultés d'évacuation différentielle de l'estomac. La quantité d'azote endogène recueillie en $24 \mathrm{~h}$ au niveau d'une canule duodénale proximale est estimée à 5,6-7,6 g par CUPERLovic, HRISTIC et ZEBrowska (I975). Plus précisément, ZkBrowska et al. (I975) en utilisant un régime protéiprive estiment à $2,2 \mathrm{~g}$ cet apport endogène d'azote au cours des $8 \mathrm{~h}$ post-prandiales. Les données de RERAT (I977) relatives à l'apparition des acides aminés dans le sang portal (donc en provenance de tout le tube digestif) après ingestion d'un régime protéiprive, permettent d'évaluer à $2,4 \mathrm{~g}$ l'azote endogène absorbé en $7 \mathrm{~h}$, encore que des variations du simple au triple soient enregistrées d'un animal à l'autre (RERAT, DUEE, I973). Globalement, l'apport endogène représenterait Io à $20 \mathrm{p}$. Ioo des protéines alimentaires selon le niveau d'ingestion (RERAT, I977).

La convergence de ces estimations conduit à retenir une valeur de l'ordre de $2 \mathrm{~g}$ pour l'apport endogène d'azote au cours des $7 \mathrm{~h}$ post-prandiales de collecte. Ce sont donc environ $80 \mathrm{p}$. roo de l'azote exogène qui devraient être évacués par l'estomac en $7 \mathrm{~h}$ après ingestion d'un repas apportant $9,9 \mathrm{~g}$ d'azote, puisque la quantité recueillie (endogène + exogène) est équivalente au total en $7 \mathrm{~h}$ à la quantité ingérée.

Toutefois, si l'on tient compte de la corrélation, durant une période plus ou moins prolongée selon le niveau d'ingestion, entre matière sèche et azote évacués par l'estomac, une tentative d'estimation de l'azote exogène récupéré, par proportionnalité à la matière sèche, peut être tentée. La différence entre ce calcul et les mesures fournirait, dans l'hypothèse d'une relation très étroite, une évaluation de l'azote endogène. Or, cette évaluation est selon les niveaux d'ingestion comprise entre 3,5 et $6,5 \mathrm{~g}$ en $7 \mathbf{h}$. Il paraît donc certain que 1'importance des quantités d'azote recueillies en $7 \mathrm{~h}$ résulte non seulement d'un apport endogène, mais aussi d'une évacuation de 1'azote relativement plus rapide que celie de la matière sèche.

\section{En conclusion}

L'évacuation gastrique de l'amidon et de l'azote chez le Porc, après ingestion d'un aliment semi-purifié à base d'amidon de maïs, présente, comme celle de la matière sèche, une phase initiale brève très intense puis un décours régulier sur un rythme plus lent. I, évacuation de l'amidon est toujours très étroitement corrélée ̀̀ celle de la matière sèche; elle apparaît comme le facteur essentiel de contrôle 
de la cinétique d'apparition des sucres réducteurs dans la veine porte. I,'évacuation apparente de l'azote, moins étroitement liée à celle de la matière sèche, s'avère beatcoup plus importante dans le même temps en raison de l'addition d'azote endogène d'une part et d'une évacuation différentielle plus rapide de l'azote, d'autre part.

Accopte pour publication en janvier 1979.

\section{Summary}

\section{Gastric emptying of starch and nitrogen in pigs ted a semi-purified diet containing maize starch}

The kinetics of starch and nitrogen gastric emptying were studied after ingestion of a semipurified diet containing $82 \mathrm{p}$. I 00 maize starch and $7 \mathrm{p}$. roo proteins in 3 pigs submitted to a reentrant fistulation of the proximal duodenum in order to collect and sample their digesta. Nitrogen was quantitatively determined according to Kjeldahl's method. Starch was measured taking into account all true glucose $(<2 \mathrm{p}$. I oo) and the $\alpha$ polymers of glucose in order to assess the relationship between gastric emptying and quantitative absorption of the hydrolysis products found in the portal vein. The method used did not at all lead to a stricto sensu quantitative determination of starch. It was developed in our laboratory by modifying Holz's method and allowed the treatment of 200 samples a day.

Gastric emptying of starch and nitrogen after a test meal supplying $800 \mathrm{~g}$ dry matter after 24 hours fasting shows like that of dry matter a short and very intense initial phase ( $23 \mathrm{p}$. I oo of the starch and $28 \mathrm{p}$. I 100 of the nitrogen ingested are emptied within ${ }_{5}$ minutes). Thereafter a regular and slower course of gastric emptying is observed. Within 7 hours, 54 p. roo of the starch and the equivalent of roo p. I oo of the ingested nitrogen were collected.

The amount of starch emptied by the stomach was always quite significantly related to that of the dry matter whatever the amount ingested. The kinetics of starch gastric emptying appeared to be the main factor controlling the kinetics of appearance of reducing sugars in the portal vein. The amount of nitrogen emptied by the stomach is less closely related to that of dry matter emitted and for a shorter time the lower the anount ingested. The relative magnitude of the apparent nitrogen emptying results both from the addition of endogenous nitrogen and from a differential and faster emptying of nitrogen.

\section{Références bibliographiques}

Argenzio R. A., Southworth M., 1975. Sites of organic acid production and absorption in gastro-intestinal tract of the pig. Am. J. Physiol., 228, 454-46o.

Borgida I. P., LAPIACF, J. P., r977. Collecte totale des contenus intestinaux chez le Porc. Automatisation de 1 échantillonnage et de la restitution à l'animal pour une étude cinétique de la digestion. Ann. Zontech., 26, 395-4I r.

Clemens E. T., Stevins C. E., Southworth M., I975. Sites of organic acid production and pattern of digesta movement in the gastro-intestinal tract of swine. $J$. Nutr., 105, 759-768.

CUBER J. C., LAP'LACE J. P., I979. Ívacuation gastrique de la matière sèche d'un régime semipurifié à base d'amidon de maïs chez le Porc. Ann. Biol. anim. Bioch. Biophys., 19, 899-905.

Cuperlovic M., Hristic V., Zebrowska T., I975. Influence of the composition of the dietary protein supplement on the digestion and absorption of protein in the small intestine of pig. Acta Veterinaria (Beograd), 25, 287-300.

Frifind D. W., Cunningham H. M., Nichot son J. W. G., ig63. The production of organic acids in the pig. 2) The effect of diet on the levels of volatile fatty acids and lactic acid in sections of the alimentary tract. Canad. J. anim. Sci., 43, I56-168.

HII, J. B., KESSLER G., I96I. An automated determination of glucose utilizing a glucose oxidase peroxidase system. J. Lab. Clin. Med., 57, 970-980. 
HOLMES J. H. G., BAYLEy H. S., HORNLY F. D., 1974. Digestion of dry and high moisture maize diets in the stomach of the pig. Br. J. Nutr., 32, 639-646.

Holz F., I977. Automatische, enzymatisch - photometrische Bestimmung des Stärkegehaltes von Cerealien. Landwirtsch. Forsch., 33, $228-249$.

KEYS J. E., de BARTHE J. V., I974, Site and extent of carbohydrate, dry matter, energy and protein digestion and the rate of passage of grain diets in swine. J. anim. Sci., 39, 57-62.

IAPLACE J. P., I978. Organisation de la motricité de l'intestin grêle chez le Porc et infiuence de 1'alimentation. Ann. Zootech., 27, 377-408.

LAPLACE J. P., Tomassone R., I970. Évacuation gastro-duodénale chez le Porc. Fistulation chronique par voie thoracique extra-pleurale; recherche d'une technique d'analyse mathématique de l'évacuation. Ann. Zootech., 19, 303-332.

Macrae J. C., Armstrong D. G., I968. Enzyme method for determination of alpha linked glucose polymers in biological materials. $J$. Sci. Food Agr., 19, 578-58r.

RERAT- A., I975a. Mesure quantitative in vivo de l'absorption chez le Porc. Application aux sucres et aux acides aminés. In : Réanimation entérale à faible débit continu. Colloque INSERM, $53,47^{-62}$.

RERAT A., I $975^{b}$. Mesure quantitative de l'absorption des glucides après ingestion d'amidon de mais chez le Porc. Med. Chir. I)ig., Sup. 2, 49-5 I.

RERAT A., 1977. Mise au point d'une méthode quantitative d'étude de l'absorption digestive chez le Porc : applications à quelques problènes nutritionnels concrets. Bull. Acad. l'et. de France, 50, 93-107.

Rerat A., Aumaitre A., Vaissade P., Vaugfiade P., I974. Mesure de l'absorption de glucides au cours de la digestion de l'amidon de maïs chez le Porc. C. R. Acad. Sci., 279 D, 83 I-834.

RFRAT A., DUEE P. H., 1973. Mesure quantitative de l'absorption des acides aminés chez le Porc recevant des régimes à taux variables de protéines. Ann. Biol. anim. Broch. Biophys., 13, $788-790$.

RERAT A., VAISSADE P., VAUGLIADE P., I978. Kinetics of absorption of some carbohydrates in the Pig. XI Int. Congr. Nutvition, Rio de Janeiro, 27 Aug.-I Sept. I 978.

Thivind P., Mercier Ch., Guilbot A., i965. Dosage de l'amidon dans les milieux complexes. Ann. Biol. anim. Bioch. Bioph:'s., 5, 513-526.

Zebrowska T., Buraczewska L., Buraczewski S., Horszczaruk F., 1975. Digestion and absorption in the small intestine of prgs. I) Digestion and absorption of dry inatter and nitrogen. Rocz. Nauk. Roln., 96 B, 79-9o. 